\title{
Investigating Learning Process Factors Related to Online Learning and Physical Examinations Among Nursing Students
}

\author{
Wanna Sanongdej ${ }^{1 *}$, Sirirat Leelacharas ${ }^{2}$, Suphamas Partiprajak ${ }^{3}$ and Kamonrat Kittipimpanon ${ }^{4}$ \\ ${ }^{1}$ Community Health Nursing Division, Ramathibodi School of Nursing, Faculty of Medicine Ramathibodi Hospital Mahidol University, Thailand \\ ${ }^{2}$ Adult and Gerontology Nursing Division, Ramathibodi School of Nursing, Faculty of Medicine Ramathibodi Hospital Mahidol University, Thailand \\ ${ }^{3}$ Community Health Nursing division, Ramathibodi School of Nursing, Faculty of Medicine Ramathibodi Hospital Mahidol University, Thailand \\ ${ }^{4}$ Community Health Nursing division, Ramathibodi School of Nursing, Faculty of Medicine Ramathibodi Hospital Mahidol University, Thailand
}

*Corresponding author: Wanna Sanongdej, Community Health Nursing Division, Ramathibodi School of Nursing, Faculty of Medicine Ramathibodi

Hospital Mahidol University, Thailand.

\begin{abstract}
Small Private Online Course (SPOC) is an online method of learning for specific group student in university. This learning method was applied in clinic for nursing students. This study sought to verify SPOC affecting the ability to perform basic physical examinations by nursing students Factors were identified following an information processing learning theory framework; these factors include online content, characteristics of online learning, online media, and brain learning processes. Data were gathered through a questionnaire survey conducted among 202 nursing students and analyzed with reference to statistics and path analysis using the software AMOS. Online characteristics, quality media online and learning processes directly influenced the physical examination ability of nursing students, whereas online content did not. Despite this, the quality media online and learning processes did influence the ability through the mediation of brain learning processes. Thus, the development of good online lessons should utilize attractive content and quality media; this will arouse the learner's brain learning processes to achieve learning purpose.
\end{abstract}

Keywords: Online course; Information processing learning theory; Basic physical examination; Nursing students

\section{Introduction}

A Small Private Online Course (SPOC) is an online course for campus, are a form of blending learning, which combine online and face-to-face instruction. Blended learning approaches provide a flexible and feasible model for student to learn [1]. Initially, SPOC was proposed by Professor Armando Fox of the University of California. It is an Effective teaching model for higher education $[2,3]$. SPOC has been adopted by University in Thailand for nursing students in clinic. This method was applied to the development of teaching and learning on the internet through the concepts of creative theories. In this method, online learning materials are usually inconsistent with topics in class. The instructor has a role in teaching media and creating online lessons and courses. The instructors prepare content, create videos, and upload these online. In addition, the teachers provide lessons, exercises, discussions, and assessments and create student tests. The instructor acts as an advisor for an online lesson while learners can study at any time and place convenient to them and repeat them if necessary. Students can follow the progress of their course, view scores, and track their own grades, and students and instructors can interact together online [2]. This study seeks to verify the paths of SPOC factors of a blended learning environment (i.e., learning using both online and traditional face-to-face classrooms). In a particular SPOC of 
University, instructors created videos covering five topics on basic physical examinations for teaching second year-nursing students. These videos for online teaching were produced by instructors to explore various health-related issues, corresponding to the different body systems including the 1) HEENT (Head-Eye-Ear-Nose-Throat), 2) lung, 3) heart, 4) abdominal, and 5) musculoskeletal systems. Before they could be used for instructional purposes, the quality and content of the online video media were checked by three media production specialists from both outside and within the university, including a media specialist and two expert nursing instructors who teach physical examinations.

Additionally, the instructors created exercises and tests on each topic for formative and summative assessments. As students study the online lessons and respond to the course by taking tests that the teacher has created, they can choose to learn following their own time, at their own place, and by choosing the content which they need to learn when they are available to go online. Students can participate in a particular lesson as many times and as long as they need. The knowledge they learn can be applied by practicing it themselves. Afterwards, students had to demonstrate to instructors in class, and lastly, they had to undergo summative tests by examining every system. The main goal of this particular SPOC is academic achievement in the preliminary examination skills of nursing students. The researcher organized the SPOC according to the information processing learning theory as a framework for teaching [4]. This theory explains that information such as visual imagery, auditory content or what the learner needs to select, and perception enters the learning process, gets stored in the shortterm memory, gets encoded, and is transferred to the long-term memory. The learner will search, link, and retrieve material stored in long-term memory [5-7]. Finally, a suitable response generation will exhibit human performance [8] in other words, the learner may reflect what they have learned. The information processing theory was applied to explain using SPOC. The SPOC is input through which the students learn process. The use of SPOC video media can serve as a stimulant with its features of image, sound, and movement, and those stimulate learning in the brain faster than letters $[5,6]$; they have been encoded and contributed to a better and longer thinking process and memorization $[8,9]$. The student can retrieve information in the memory to link previous knowledge with new knowledge. The outcome of this is basic examination content. The nursing students' academic achievement in performance skills is a primary goal of this SPOC.

SPOC is a course online learning modality which is set a restricted-access course for specific group student in university $[1,2]$. As online learning has emerged as a relatively new and growing alternative to the traditional face-to-face classroom learning, which has gained prominence as an alternative learning method during the current COVID-19. There are a number of studies that have examined the results or the accomplishments of online learning $[2,3,5]$. Despite this, many have still not explored online learning factors through the use of direct and indirect analysis of pathway effects. Indeed, analysis of the pathway is necessary as it can explain the reasons for achieving analytical results. The results can serve to help improve teaching plans to enhance the skills of nursing students and to further improve nursing curricula.

Aim and hypothesis. This study aims to verify the effects of relationships among the variables used in the theoretical model of online lessons, based on information processing learning theories. It seeks to identify the direct and indirect effects of the selected factors, which includes online content, quality online media, online characteristics, and learning process, that affect basic physical examination performance.

\section{Conceptual Framework and Theoretical Model}

Based on the introduction above, we created a framework as summarized in Figure 1.

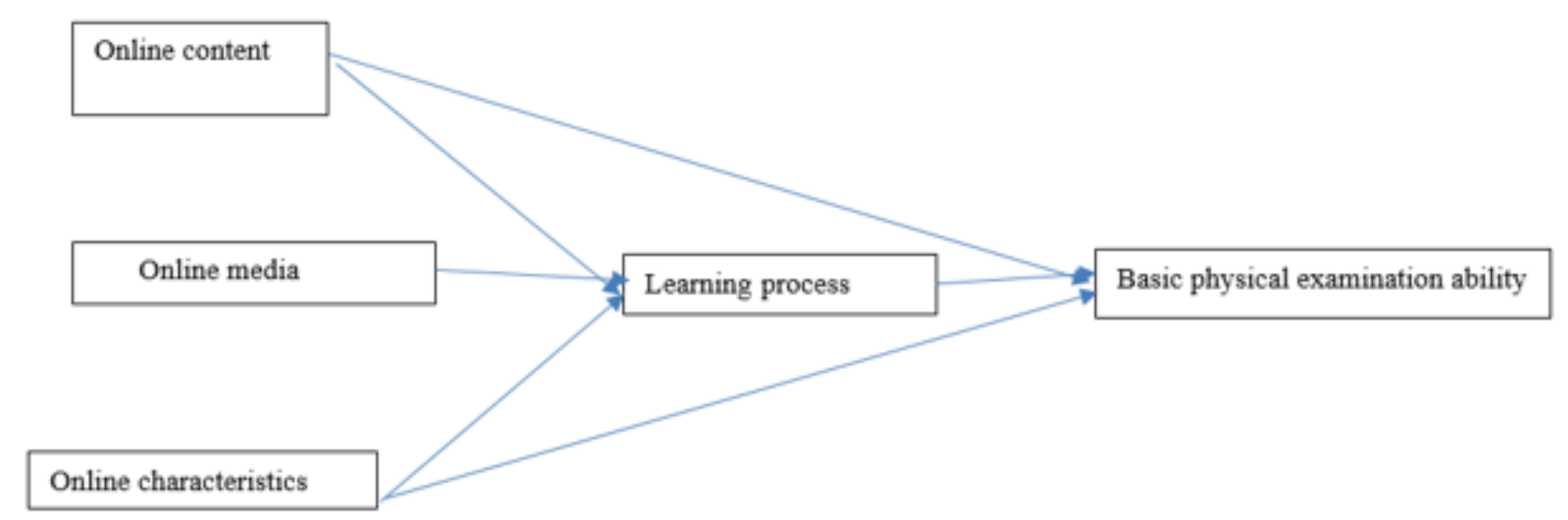

Figure 1: Conceptual Framework of the Current Study. 


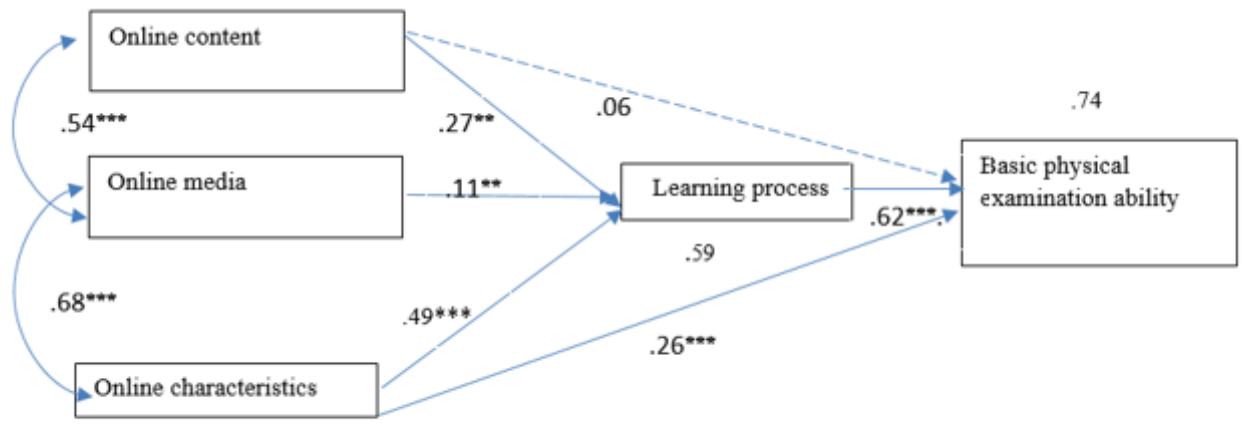

Figure 2: Model learning process of basic physical examination ability.

Statistical variance $\times 2=699.18$, df 10, $p=.686$, RMSEA $=.00$, RMR .013, GFI 1.00, CFI .995. All paths shown are statistically significant $(p<$ $.05, p<.05, p<.001)$ except for online content.

\section{The conceptual framework}

For this study, information processing theories served as our framework [4]. This is a learning process model that can be used to describe learning as being composed of storage in short memory, encoding, and transfer to long-term memory. In other words, the learner will search, link, and retrieve material stored in the longterm memory to perform accurately. Initially, a learner would stimulate the environment which would affect a learner's nervous system or learning process. A new input item, such as an image, letter, number, a single-syllable word, or sound, will stimulate learners. Then, learners become interested due to the stimulant and select what they need to learn. This process is called "selection perception." The learning process occurs among learners will select what they need to learn [4]. Next, the learner will select what he needs to learn. The clue will be an image or a sound. The learner will establish content as content features in the field of hearing, or as a visual image in the learner's nervous system as part of shortterm memory. Moreover, receiving the same exciting information repeatedly will input it in short-term memory. A process called rehearsal will help the semantic encoding of information into the long-term memory. The memory processes are stored in the learner's nervous system. When external stimuli or events influence learners, they will search, retrieve the pre-coded cues, and recall them to respond via a specific performance. Learners will execute an action and/or a skilled movement sequence.

The information processing theory and its account of memory is adopted here to explain the use of SPOC. A SPOC is a form of e-learning; it is an information delivery method which puts the learning process online for students. The nursing students' academic achievement regarding their performance skills is the primary goal of the particular SPOC by the university. There are several factors that can be delivered by SPOC, including online content, media, and their characteristics.
The content from online lessons is what learners need to receive or select to receive, affect the thinking process, and memorize. Contents refer to facts, concepts, theories, in specific academic courses, and skills. They make learners understand lessons and able to perform basic examinations. The content of an online lesson should be clear, concise, accurate, and organized from easy to difficult [10]. Video media used in teaching via SPOC make use of images, sound, and movement, which stimulate learning processes in the brain faster than letters do [11]. Image and moving images in particular are excellent features that positively affect the learning process [11]. Sound will stimulate the brain regions involved in auditory-motor coupling and address the role of mechanisms such as auditory-motor entrainment, auditory mirror neurons, and sensorimotor integration [12]. Sound has been found to improve motor perception, leading to enhanced learning performance and memory $[7,12]$. Online instruction is an excellent method for delivering content for nursing students. A characteristic of online teaching is that learners are able to access the lesson whenever it is convenient. The teacher and student can increasingly interact and discuss lesson material, and the students can repeat lessons until they understand the material, and this facilitates the learning process. In this way online lessons can increase a learner's knowledge and capacities $[9,13]$.

\section{Materials and Methods}

This study was cross-sectional in design. The study's overall purpose was to confirm path way relationships of selected factors, including online content, the quality of the online media, and online characteristics, all of which may affect basic physical examination performance. Also, the learning process was selected as a mediator in the relationship between selected factors and basic examination performance ability.

\section{Ethical considerations}

This study was approved to ethical consideration from the 
Ethics Committee on Human Right related to Research Involving Human Subjects of the Faculty of hospital, University. (No. IRB MURA2019/727). The second-year nursing students were informed about details of the study, and they were invited to participate. The participation was voluntary and they were assured of confidentially and anonymity in the use of data. Written informed consent was obtained from every participant.

\section{Participants}

The study population were second-year nursing students studying for a bachelor's degree at a nursing school in [blinded] Hospital, [blinded] University. The purposive sample included second-year nursing students who had been attending a health assessment course via a SPOC. According to statistics, the best sample for our study design should be 20 times the number of parameters (i.e., 20 x 5 in our study) in the path analysis [4]; thus, a sample size of 100 would have been enough for a path analysis using the software AMOS. However, 202 participants were used for analysis in this study, so this strengthens the study. Most of the participants were women (95\%) and between 20 to 24 years old with $45.05 \%$ studying each online lesson twice (i.e., the five different videos, twice)

\section{Data collection}

Online media were developed by researcher's team in 2017 These were examined for valid content and quality by three experts from University before its use was permitted in learning as part of the health assessment course. Data was collected from 1 September to 30 October 2019 at the Nursing School of University in the Salaya region of Thailand. The researcher informed the nursing students about the details of the present study. After students completed the health assessment course a basic physical examination demonstration with their instructors. Two hundred and twenty questionnaires were distributed, and 210 were returned; eight questionnaires had incomplete data so only 202 were used for the final analysis.

\section{Questionnaires}

Questionnaire items were derived from interviews and a review of the literature. The content index was qualified by five experts, including two media experts and the three nursing instructors who were competent in physical examinations. Questionnaires were trialed using 30 nursing students and a total of 205 questions required for construct validity. Six self-administered questionnaires were used to obtain data: the Demographic Data Form (DDF), the Online Content Questionnaire (OCQ), the Quality of Media Questionnaire (QMQ), Characteristics of Online Lessons (COL), the Learning Process (LP), and the Physical Examination Ability Questionnaire (PEAQ). The Demographic Questionnaire was used to obtain the demographic information of the senior nursing students; information gathered included sex, age, number of times studying online, and online entry study period each time.

The OCQ is composed of seven items used to identify specific online content, including content in accordance with the objectives, accuracy, and reliability of content, sorting content from easy to learn, then manageable, then extremely difficult to learn. Each item is rated on a 4-point Likert scale ranging from 1 (not agree) to 4 (strongly agree), with a higher score indicating excellent online content. The questionnaire's content validity index I-(CVI) was .831, and S-CVI .79. The individual construct reliability of items was .53-.63. The Cronbach's alpha reliability was .77. The QMQ consists of four items. Questions were used to measure the quality of media, including the quality of sound, light, clarity, and letters. Each item is rated on a 4-point Likert scale ranging from 1 (strongly disagree) to 4 (strongly agree), with a higher score indicating an outstanding quality of media. The questionnaire's content validity index (CVI) was .78-.83, and S-CVI .96. The individual construct reliability of items was .42-.78, and the Cronbach's alpha reliability was .73.

The COL was used to measure the characteristics of online lessons. Its six items are measured on a 4-point Likert rating scale ranging from 1 (strongly disagree) to 5 (strongly agree). The survey assesses the students' perception of characteristics of online lessons in terms of quality of convenience to access, control time, determination of place, links to other web sites, and interaction with the instructor. The questionnaire's CVI was 0.831- and S-CVI .77 , the individual construct reliability of items was $0.420-.78$, and the Cronbach's alpha reliability was 0.87 . The LP is a 5 -item questionnaire. Characteristics of online lessons were developed following information processing theories and memory. Items were used to measure stimulating curiosity, extracting images from thought, ease of remembering pictures, longer retention in memory, and enjoyment of learning. Each item is rated on a 4-point Likert scale ranging from 1 (strongly disagree) to 4 (strongly agree). The total possible score obtainable ranges from 0 to 24 , with a higher score indicating a higher learning process stimulation. The questionnaire's CVI was .49-.82, the individual construct reliability of items was .48-.88, and the Cronbach's alpha reliability was .83. The PEA is a 5-item questionnaire that measures a nursing students' perceptions of their physical examination ability. Items are rated on a 4-point Ffrating scale ranging from 0 (strongly disagree) to 4 (strongly agree), assessing better physical examination skills, increased physical examination capabilities, and making physical examination easier. The total possible score ranges from 0 to 20, with higher scores indicating a higher perceived ability to perform basic physical examinations. The CVI was .83, and S-CVI .97. The individual items had a construct reliability of 0.520-.89. The Cronbach alpha reliability was .84 .

\section{Statistical analysis}

For the descriptive statistics of participants, we used frequency, percentage, mean, standard deviation, and SPSS software version 
24.0 to examine the range of scores. The research instruments were analyzed for reliability and construct validity using the AMOS 24.0 software. The assumption of path analysis, including normality, multicollinearity, linearity, and homoscedasticity were examined using SPSS. To analyze the influence of online teaching affecting Table 1: Personal Information of Senior Nursing Students. the ability to perform basic physical examinations, we used Path Analysis Statistics, statistical maximum likelihood estimation (MLE) of AMOS. The model was verified based on $\chi 2, \chi 2 / d f$, the goodness of fit index (GFI), comparative fit index (CFI), and root mean square error of approximation (RMSEA) (Table 1-4).

\begin{tabular}{|c|c|c|}
\hline Variable & Number & Percent \\
\hline \multicolumn{3}{|l|}{ Sex } \\
\hline Female & 187 & 92.57 \\
\hline Male & 7 & 7.43 \\
\hline \multicolumn{3}{|l|}{ Age range } \\
\hline $20-24$ & 202 & 100 \\
\hline \multicolumn{3}{|c|}{ Average 22.5 years, SD 1.2} \\
\hline \multicolumn{3}{|c|}{ Number of entering SPOC per issue (times) } \\
\hline 1 & 37 & 18.31 \\
\hline 2 & 91 & 45.05 \\
\hline 3 & 58 & 28.72 \\
\hline 4 & 12 & 5.94 \\
\hline 5 & 2 & 0.99 \\
\hline 6 & 2 & 0.99 \\
\hline \multicolumn{3}{|c|}{ Period of entering SPOC per issue (min) } \\
\hline$<10$ & 5 & 2.47 \\
\hline $10-30$ & 60 & 29.7 \\
\hline $31-60$ & 120 & 59.41 \\
\hline$>60$ & 17 & 8.42 \\
\hline \multicolumn{3}{|c|}{ Mean = $43.50 \mathrm{~min}$} \\
\hline \multicolumn{3}{|c|}{$\mathrm{SD}=10.25 \mathrm{~min}$} \\
\hline
\end{tabular}

Table 2: Descriptive Statistics of Study Variables $(n=202)$.

\begin{tabular}{|c|c|c|c|c|}
\hline Variable & Possible Range & Actual Range & Mean & SD \\
\hline Online content & $0-28$ & $15-28$ & 24.94 & 2.59 \\
\hline Online media & $0-16$ & $9-16$ & 13.56 & 1.91 \\
\hline Online characteristics & $0-28$ & $7-28$ & 23.53 & 3.32 \\
\hline Learning process & $0-24$ & $6-24$ & 20.34 & 3.21 \\
\hline Basic physical examination ability & $0-20$ & $15-28$ & 24.94 & 2.59 \\
\hline
\end{tabular}

Table 3: Standardized Coefficients and Unstandardized Coefficients.

\begin{tabular}{|c|c|c|}
\hline Variable & Standardized Coefficient & Unstandardized Coefficient \\
\hline Online content $\rightarrow$ Learning process & 0.27 & 0.33 \\
\hline Online media $\rightarrow$ Learning process & 0.11 & 0.18 \\
\hline Online characteristics $\rightarrow$ Learning process & 0.49 & 0.47 \\
\hline Learning process $\rightarrow$ Basic physical examination ability & 0.67 & 0.61 \\
\hline Online content $\rightarrow$ Basic physical examination ability & 0.06 & 0.06 \\
\hline Online characteristics $\rightarrow$ Basic physical examination ability & 0.26 & 0.25 \\
\hline Quality online media $\rightarrow$ Basic physical examination ability & 0.27 & 0.2 \\
\hline
\end{tabular}


Table 4: The Results of the Influence of Online Teaching.

\begin{tabular}{|c|c|c|c|c|c|c|}
\hline \multirow{2}{*}{ Causal Variance } & \multicolumn{3}{|c|}{ Learning Process } & \multicolumn{3}{|c|}{ Physical Examination Ability } \\
\hline & TE & IE & DE & TE & IE & DE \\
\hline Online content & $.27^{* *}$ & - & $.27^{* *}$ & $.22 *$ & $.16^{*}$ & 0.06 \\
\hline Quality of media & $.11^{*}$ & - & $.11^{*}$ & $.086^{* *}$ & $.65^{* *}$ & $.20^{*}$ \\
\hline Online characteristics & $.49 * *$ & - & $.49^{* *}$ & $.556^{*}$ & 0.295 & $.251^{*}$ \\
\hline Learning process & - & & & $.607^{* *}$ & - & $.607^{* *}$ \\
\hline \multicolumn{7}{|c|}{ Statistical variance $\times 2=699.18, \mathrm{df} 10, \mathrm{p}=.686, \mathrm{RMSEA}=.00, \mathrm{RMR} .013, \mathrm{GFI} 1.00$, AGFI .995} \\
\hline Variables & \multicolumn{3}{|c|}{ Learning process } & \multicolumn{3}{|c|}{ Basic physical examination ability } \\
\hline $\mathrm{R} 2$ & \multicolumn{3}{|c|}{$59 \%$} & \multicolumn{3}{|c|}{$74 \%$} \\
\hline
\end{tabular}

\section{Results}

The results showed how the different measured parameters affected the ability to perform basic physical examination among nursing students. The hypothesized model is in accordance with real data which was collected. $\chi 2=699.18$, df $10, p=.686$, RMSEA $=.00$, RMR .013, GFI 1.00, CFI .995. When researchers considered three factors, including online content, online media quality, and online characteristics, it was shown that online characteristics could affect basic physical examinations and this relationship was statistically significant $(.262, \mathrm{p}<.001)$, and could affect the learning process, and this relationship was also statistically significant (.49, $\mathrm{p}<.001$ ). In contrast, online content had no direct effect on the basic physical examination ability of nursing students (.06, $p>.05$ ) The learning process as a mediator, had a substantial immediate impact on nursing students' initial physical examination ability $(.62, \mathrm{p}<.001)$.

When considering indirect effects, it was shown that there was an indirect influence of ability on basic physical examination Quality online media, and online characteristics had an indirect impact on basic physical examination through the learning process. The causal relationships of Quality of media, Online content, and Online characteristics and, Learning process accounted for $74 \%$ of the basic physical examination ability. The explained variance in the learning process was $59 \%$. The learning process mediated the relationship between Quality of media, Online content, Online characteristics and Basic physical examination ability. The results of the model testing are shown in Figure 2 and Table 3, 4.

\section{Discussion}

Online lessons have a direct and indirect influence on the ability to perform basic physical examinations. The online lesson features are easily accessible, convenient, and quick to access These make the learners comfortable in choosing when they are ready and intend to participate. All these factors can make learners more open-minded and willing to learn. Therefore, the result of online instruction is more effective outcomes. Online content was an important variable for our model because many studies had indicated that well-designed course content is an indication for developing effective online instruction. The present study found that content had an indirect effect but not a direct impact on the nursing practice in question. This was inconsistent with wherein nursing students had their knowledge scores and competency increase significantly after studying via an online course. [12] For this study, it can be explained by information processing theories that although content can be correct and accurate, graded from easy to difficult, consistent with the objectives, these may not make learners remember enough to put what they learn into practice. Therefore, content must be transmitted via an excellent medium through thinking processes, resulting in effective learning. The content must go through the learning process via stimuli and the memory. Through this, proper knowledge that can affect one's performance will occur.

Media had an indirect effect on the nursing practice. Several studies show that light, sound, and pictures affect the brain's learning process and emotions [12-16]. These will stimulate the brain, resulting in better recognition ability compared to letters. The results of this study showed that skills in nursing practice should be developed. Teachers need to create teaching materials that stimulate the brain to make learning more fun for students. Stimulating visuals will lead to remembering images for a longer time, resulting in more extended practice skills. Therefore, the development of good teaching media should stimulate the learning system of the brain, leading to effective learning. The teaching content and media properties had no direct influence on learning. This means they did not evoke interest among students, students would not be likely to extract prior images and remember.

The participants of this study were selected using nonprobability sampling. Most of them were female: thus, the homogenous samples might not offer adequate information to represent nursing students generally. The findings from this study may not be generalizable to all nursing students. The theoretical model might be further tested among in a range of groups (different class, type of education) or test learners before and after online courses. 


\section{Conclusion}

This study was conducted to confirm learning process factor to affect the ability to perform basic physical examinations among nursing students. The results of the study showed the online characteristics, online media, and learning processes have a direct and indirect influence on the ability to conduct physical examinations among nursing students, though excluding online content. Online media and online characteristics influenced learning by stimulating the learning system. Only online content did not directly improve learners' performance. Thus, the development of good online lessons should utilize content which stimulates the learning process. Content must be attractive to learners and use methods that will arouse the learner's interest in specific learning and extract prior images from memory. This should be done; the results of the study could be applied more generally.

\section{Acknowledgement}

I would like to express my sincere thanks to Ramathibodi School of Nursing, Faculty of Medicine Ramathibodi Hospital for all theirs support throughout the period of this study.

\section{Conflict of Interest}

No conflict of interest for this study.

\section{References}

1. Cheng HNH, Liu Z, Sun J, Liu S, Yang Z (2017) Unfolding online learning behavioral patterns and their temporal changes of college students in SPOCs. Interactive Learning Environments 25(2): 176-188.

2. Guo P (2017) MOOC and SPOC, which one is better? EURASIA J Math Sci and Tech Ed 13(8):5961-5967.

3. Means B, Toyama Y, Murphy R, Bakia M, Jones K (2009) Evaluation of evidence-based practices in online learning: A meta- analysis and review of online learning studies. http://www2.ed.gov/rschstat/eval/tech/ evidence-based-practices/finalreport.pdf.
4. McLeod S A (2008) Information processing. Simply Psychology. https:// www.simplypsychology.org/information-processing.htm.

5. McDaniel MA, Einstein GO (1986) Bizarre imagery as an effective memory aid: The importance of distinctiveness. Journal of Experimental Psychology: Learning, Memory, and Cognition 12 (1): 54-65.

6. Davis T, Xue G, Love BC, Preston AR, Poldrack RA (2014) Global Neural Pattern Similarity as a Common Basis for Categorization and Recognition Memory. The Journal of Neuroscience 34(22): 7472-7484.

7. Sweeten TL (2017) Mnemonic Mechanisms for Making Memories. Journal on Empowering Teaching Excellence: 1(2).

8. Patton WW (1991) Opening students' eyes: Visual learning theory in the Socratic classroom. Law and Psychology Review 15: 1-18.

9. Al-Imamy S, Alizadeh J, Nour MA (2006) On the Development of a Programming Teaching Tool: The Effect of Teaching by Templates on the learning process. Journal of Information Technology Education 5(1): 271-283.

10. Hjorth JE, Hofoss D, Kynø NM (2019) E-learning or lectures to increase knowledge about congenital heart disease in infants: A comparative interventional study. Nursing Open 6: 1143-1149.

11. Shabiralyani G, Hasan KS, Hamad N, Iqbal N (2015) Impact of Visual Aids in Enhancing the Learning Process Case Research: District Dera Ghazi Khan. Journal of Education and Practice 6(19): 226-234.

12. Hori S, Mori K, Machimo T, Saimaya A (2017) Effects of Light and Sound on the Prefrontal Cortex Activation and Emotional Function: A Functional Near-Infrared Spectroscopy Study Front. Frontier in Neuroscience 11: 321.

13. Martin-Blas T, Serrano-Fernandaz A (2009) The role of new technliogies in learning process: Model as a teaching tool in Physics. Computers \& Education 52(1): 35-44.

14. Wright S (1980) Path analysis. https://www.statisticssolutions.com/ path-analysis/.

15. Kuzovkin I, Vicente R, Petton M, Lachaux JP, Baciu M, et al. (2018) Activations of deep convolutional neural networks are aligned with gamma band activity of human visual cortex. Communications Biology 1: 107 .

16. Anderson DR, Kirkarian HL (2015) Media and cognitive development Handbook of child psychology and developmental science (2)22: 1-46. 